\title{
Atlas-Based Reduced Models of Blood Flows for Fast Patient-Specific Simulations
}

\author{
K. McLeod ${ }^{1}$, A. Caiazzo ${ }^{2}$, M.A. Fernández ${ }^{2}$, T. Mansi ${ }^{1}$, \\ I.E. Vignon-Clementel ${ }^{2}$, M. Sermesant ${ }^{1}$, X. Pennec ${ }^{1}$, Y. Boudjemline ${ }^{3}$, and \\ J.F. Gerbeau ${ }^{2}$ \\ 1 INRIA Méditerranée, Asclepios Project, Sophia Antipolis, France \\ ${ }^{2}$ INRIA Rocquencourt, REO Project, Rocquencourt, France \\ 3 Service de Cardiologie Pédiatrique, Hôpital Necker-Enfants Malades, Paris, France
}

\begin{abstract}
Model-based interpretation of the complex clinical data now available (shape, motion, flow) can provide quantitative information for diagnosis as well as predictions. However such models can be extremely time consuming, which does not always fit with the clinical time constraints. The aim of this work is to propose a model reduction technique to perform faster patient-specific simulations with prior knowledge built from simulations on an average anatomy. Rather than simulating a full fluid problem on individual patients, we create a representative 'template' of the artery shape. A full flow simulation is carried out only on this template, and a reduced model is built from the results. Then this reduced model can be transported to the individual geometries, allowing faster computational analysis. Here we propose a preliminary validation of this idea. A well-posed framework based on currents representation of shapes is used to create an unbiased template of the pulmonary artery for 4 patients with Tetralogy of Fallot. Then, a reduced computational fluid dynamics model is built on this template. Finally, we demonstrate that this reduced model can represent a specific patient simulation ${ }^{4}$.
\end{abstract}

\section{Introduction}

When considering a large set of patient geometries, full flow simulations can be rather costly if they need to be carried out individually on each patient. However, often relevant information about the fluid solution can be extracted from reduced models. We present a method which combines an atlas-based technique and model reduction approaches, in order to create a database of pre-computed flow characteristics, which enables fast patient-specific flow simulations. This work investigates the validity of applying reduced models and atlas-based methods to decrease flow computational cost, and in particular to examine under which conditions these methods can be useful for obtaining clinically relevant features.

Let us assume to be given a set of individual patient geometries. We construct a template (also called an 'atlas') of the pulmonary artery based on the

\footnotetext{
${ }^{4}$ Published in Lecture Notes in Computer Science, Vol. 6364, p. 95-104, 2010
} 
"currents" approach, as described in detail in [1,2]. In [1], a "forward" model is proposed where the set of shapes (in this case surfaces) are considered as random deformations of an unknown "ideal" template plus some residuals. A key advantage of this framework is that the template is unbiased. Our idea is to perform a full fluid simulation, later called a direct simulation, only on an atlas of the population. Results can be used to extract a reduced model which, opportunely mapped back onto particular geometries, can be used to perform faster reduced simulations. In particular, the reduced model is based on a Proper Orthogonal Decomposition (POD) of the fluid solution, i.e. a description of the flow field in a lower dimensional space, with a considerably lower number of degrees of freedom.

To illustrate the approach, we applied this method to Tetralogy of Fallot $(\mathrm{ToF})$ patients. ToF is a severe congenital heart defect that requires open heart surgical repair within the first year of life. Understanding the shape of the pulmonary artery and the flow of blood through the artery is crucial for follow-up treatment planning. A fast and effective method to retrieve relevant information about the patient-specific blood flow in the pulmonary artery of ToF patients is motivated by the need of assisting cardiologists in determining the optimal placement and size for valve replacement. But CFD simulations are known to be very time consuming. A model reduction approach could therefore be useful in this context.

Here, considering a data set of 4 ToF patients, we present a preliminary $a$ posteriori validation of the model reduction method, comparing the full solution on individual patients with its projection on the POD basis mapped from the template.

\section{Model Reduction with Atlas-Based Methods}

The pulmonary artery of each patient was segmented from MR angiography as described in Sec. 3.1. Initially, an unbiased template of the artery shape was constructed to serve as a reference atlas for the population.

Blood flow inside the template was simulated numerically, and a proper orthogonal decomposition (POD) of the solution was derived. This decomposition, transported to the individual geometries, may allow to reduce the computational expenses of individual flow simulations. This method is summarized in fig. 1.

\subsection{Unbiased Template of the Pulmonary Artery in ToF Patients}

The pulmonary artery template is created using the forward strategy proposed in [1]. This approach is particularly suited for our purposes as it is non-parametric since shapes are represented by currents, so that the framework can be used for meshes that initially have no point correspondences. However, when the template is mapped to an individual, a one-to-one correspondence between points is established between the template and the mapped individual, which is a useful 


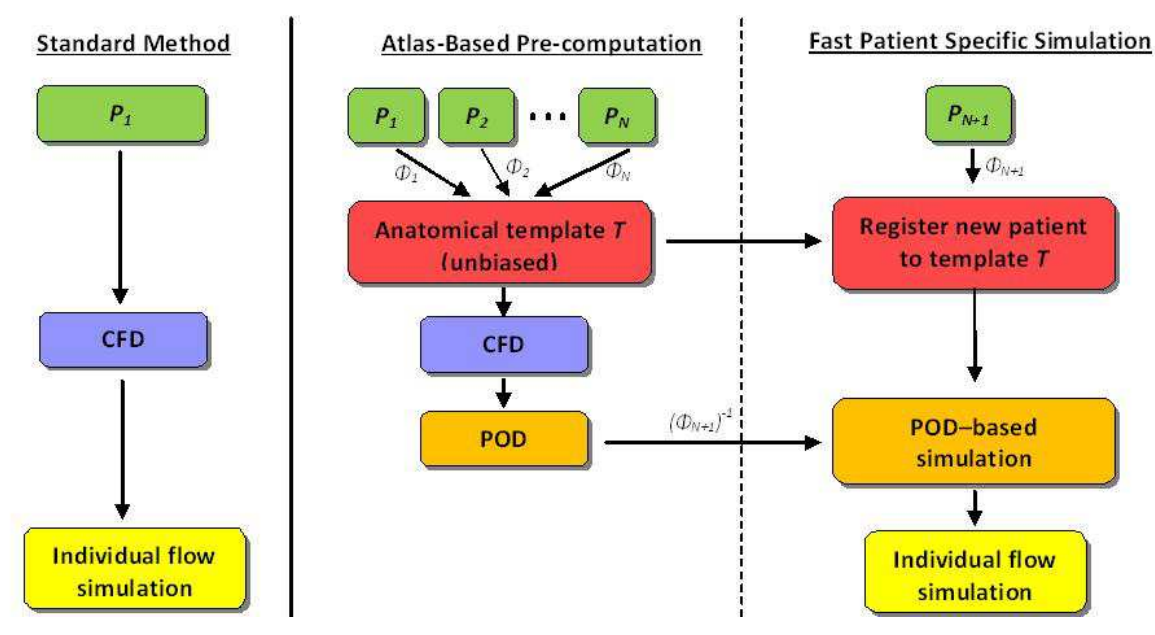

Fig. 1. Left column: the standard method for individual fluid flow simulation. Centre column: pre-computed Computational Fluid Dynamic (CFD) simulation using atlasbased methods, with model reduction using Proper Orthogonal Decomposition (POD). Right column: using the pre-computed fluid simulation and model reduction, fluid simulation can be applied to a new patient quickly, by first registering the template to the patient, then deforming the POD modes using the inverse of this deformation.

feature for later applying reduced models that necessitate such a parameterization. Also, the framework has the advantage in that the template is unbiased with respect to the population used to create it. What this means is that adding new patients drawn from the same population at the template creation step will have little effect to the final template.

The pulmonary artery surfaces $\mathcal{T}^{i}$, or shapes, are modelled as the sum of a diffeomorphic deformation $\varphi^{i}$ of the template $\widehat{\mathcal{T}}$ and a residual term $\epsilon^{i}$ standing for the shape features that cannot be represented by the template (topology changes, acquisition artefacts, etc.): $\mathcal{T}^{i}=\varphi^{i}(\widehat{\mathcal{T}})+\epsilon^{i}$. Currents are used to represent the shapes, the residuals and the deformations in the same common framework. They enable the usual operations (mean, variance...) on shapes as they form a vector space. Intuitively, currents can be seen as objects that give the flux of any vector field $\omega \in W$ across the shapes. $W$ is a Reproducing Kernel Hilbert Space, RKHS, of infinite dimension generated by a Gaussian kernel $K_{W}(\mathbf{x}, \mathbf{y})=\exp \left(-\|\mathbf{x}-\mathbf{y}\|^{2} / \lambda_{W}^{2}\right)$ that defines an inner product in $W$ (and also in the dual space of $\left.W, W^{*}\right)$. $W^{*}$ is therefore a vector space of linear mappings from $W$ to $\mathbb{R}$, which is defined as the space of currents.

More precisely, a triangle centred at $\mathbf{x}$ with normal $\alpha$ is represented by the Dirac delta current $\delta_{\mathbf{x}}^{\alpha}$. Therefore, a discrete mesh is encoded by the sum of the currents of its triangles $\mathcal{T}^{i}=\sum_{k} \delta_{\mathbf{x}_{k}^{i}}^{\alpha_{k}^{i}}$. The residuals $\epsilon^{i}$ are modelled as a Gaussian distribution on the $\alpha_{k}^{i}$. The deformation $\varphi^{i}$ that registers the template 
$\widehat{\mathcal{T}}$ to the current $\mathcal{T}^{i}$ is estimated using the Large Deformation Diffeomorphic Mappings (LDDMM) framework [3]. $\varphi^{i}$ is parameterised by a smooth initial vector speed $\mathbf{v}_{0}^{i}$, which also belongs to a Gaussian RKHS $V$ with variance $\lambda_{V}^{2}$. Moreover, this initial speed vector field is completely defined by the moment vectors $\beta^{i}$ centred at the same point location as the template moments: $\mathbf{v}_{0}^{i}(\mathbf{x})=$ $\sum_{k} K_{V}\left(\mathbf{x}_{k}, \mathbf{x}\right) \beta_{0}^{i}\left(\mathbf{x}_{k}\right)$. Finally, the template $\widehat{\mathcal{T}}$ and the deformations $\varphi^{i}$ towards each patient are estimated by means of an alternate two-step strategy, initialised with the mean current of the population.

\subsection{Blood Flow Simulation on the Reference Geometry}

Let us denote with $\widehat{\Omega}$ the three dimensional domain defined by the template surface $\widehat{\mathcal{T}}$. This domain is discretized using a tetrahedral mesh $\widehat{\Omega}_{h}(h$ being the level of refinement of this discretization, see fig. 2, right). Blood flow is simulated solving numerically the incompressible Navier-Stokes equations with a Lagrangian P1 finite element (FE) formulation. In the following, $\left(\widehat{v}_{1}, \ldots, \widehat{v}_{M}\right)$ denotes the finite element basis, and $\left(\widehat{\mathbf{u}}_{h}, \widehat{p}_{h}\right)$ denote the velocity and pressure fields computed on the reference domain. For example, the pressure field is decomposed as $\widehat{p}_{h}(x)=\sum_{j=1}^{M} p_{j} \widehat{v}_{j}(x)$, where $p_{j}$ is the value of $p_{h}$ at node $j$. A similar decomposition is used for the velocity.

A flow rate is prescribed at the inlet, with a mean value of $4.2 \mathrm{~L} \cdot \mathrm{min}^{-1}$. At the outlet a Windkessel model (zero-dimensional model) was coupled to the three-dimensional model to represent the impedance of the distal pulmonary vasculature [4]. Values of the proximal resistance, capacitance and distal resistance are chosen so that the average pressure is $10 \mathrm{mmHg}$ and that the pressure ranges from $0 \mathrm{mmHg}$ to $25 \mathrm{mmHg}$ (since at this stage, these patients have no functioning valve).

Proper Orthogonal Decomposition (POD) A proper orthogonal decomposition (POD) of a numerical solution (or, in general, of a given set of data, see, e.g., [5-7]) consists of finding a set of basis functions (orthogonal w.r.t. a given scalar product) which, even containing a small number of elements, can represent sufficiently well the numerical solution. This approach, besides reducing the data size without losing relevant information, allows to perform faster numerical simulations, by restricting the space of the solution to the subspace generated by the POD basis functions.

We computed a POD basis $\left\{\widehat{\boldsymbol{\phi}}_{i}^{\mathbf{u}}, \widehat{\phi}_{i}^{p}\right\}_{i=1}^{N_{\mathrm{m}}}$ containing $N_{\mathrm{m}}$ modes, with $\widehat{\boldsymbol{\phi}}_{i}^{\mathbf{u}}:$ $\widehat{\Omega} \rightarrow \mathbb{R}^{3}$ (velocity POD basis functions) and $\widehat{\phi}_{i}^{p}: \widehat{\Omega} \rightarrow \mathbb{R}$ (pressure POD basis functions), for $i=1, \ldots, N_{\mathrm{m}}$. For example, a pressure POD basis function $\widehat{\phi}_{i}^{p}$ is known through its decomposition on the finite element basis: $\widehat{\phi}_{i}^{p}(x)=$ $\sum_{j=1}^{M} \phi_{i, j}^{p} v_{j}(x)$, where $\phi_{i, j}^{p}$ denotes the value of $\phi_{i}^{p}$ at node $j$.

\subsection{Mapping POD on Individual Geometries}

POD provides a reduced description of the flow solution on the template shape. In order to obtain in further studies a suitable representation of the flow in 
individual patient geometries, with a reduced computational cost, our aim is to transport the template POD on the individual geometry.

Deformation of the Reference Domain To fix the notation, let us denote with $\Omega$ a particular patient geometry, and with $\mathcal{T}$ its external surface. As for the template, we will call $\Omega_{h}$ and $\mathcal{T}_{h}$ the discretizations of, respectively, $\Omega$ and $\mathcal{T}$ (see fig. 2, right). We consider the individual geometry as a deformation of the template, assuming to be given a deformation map

$$
\mathcal{A}: \widehat{\Omega} \rightarrow \Omega
$$

which maps the reference template onto the individual (deformed) domain. Let $F=\nabla \mathcal{A}$ be the deformation gradient and $J=\operatorname{det} F$ its Jacobian.

Reference POD Transported to Individual Geometries Through $\mathcal{A}$ we can define a change of variables between the template $\widehat{\Omega}$ and the particular geometry $\Omega$. Hence, the transported pressure POD basis function $\phi_{i}^{p}$ is defined as

$$
\phi_{i}^{p}(\mathbf{x})=\widehat{\phi}_{i}^{p}\left(\mathcal{A}^{-1}(\mathbf{x})\right)
$$

for all $\mathbf{x} \in \Omega$. Yet, for solenoidal vector fields like $\widehat{\phi}_{i}^{\mathbf{u}}$, the simple change of variables (2) is no longer appropriate. Instead, following [8], we transport the velocity POD basis using the relation

$$
\phi_{i}^{\mathbf{u}}(\mathbf{x})=\frac{1}{\left|J\left(\mathcal{A}^{-1}(\mathbf{x})\right)\right|} F\left(\mathcal{A}^{-1}(\mathbf{x})\right) \widehat{\phi}_{i}^{\mathbf{u}}\left(\mathcal{A}^{-1}(\mathbf{x})\right)
$$

for all $\mathbf{x} \in \Omega$. Equation (3) is known as the (inverse) Piola transform (see, e.g., [9]). It has the remarkable property that the transform of a divergence free vector field, in $\widehat{\Omega}$, is also divergence free, in $\Omega$.

Computation of the deformation gradient In practice, information about geometries is only based on a discretization of the surfaces. However, knowing $\left.\mathcal{A}\right|_{\mathcal{T}}$, even if only on a discretization of the surface, will be sufficient for our purposes. Indeed, in case of moderate deformations, the map $\mathcal{A}$ in the complete volume can be generated as a harmonic extension of $\left.\mathcal{A}\right|_{\mathcal{T}}$. Next, from the discrete form of $\mathcal{A}$, one can compute an approximation $F_{h}$ of the deformation gradient,

$$
F_{h}^{\alpha \beta}(\widehat{\mathbf{x}})=\sum_{i=1}^{M} F_{i}^{\alpha \beta} \widehat{v}_{i}(\widehat{\mathbf{x}})
$$

by projecting (for the $L^{2}$ norm) $\nabla \mathcal{A}$ on the finite element basis. This can be done solving the linear systems

$$
\widehat{M}\left[F_{i}^{\alpha \beta}\right]_{i=1, \ldots, M}=\left[\int_{\widehat{\Omega}} \partial_{\beta} \mathcal{A}_{\alpha}(\widehat{\mathbf{x}}) \widehat{v}_{i}(\widehat{\mathbf{x}}) \mathrm{d} \widehat{\mathbf{x}}\right]_{i=1, \ldots, M},
$$


for each coordinate $\alpha, \beta=x, y, z$, with $\widehat{M}=\left[\int_{\widehat{\Omega}} \widehat{v}_{i} \widehat{v}_{j}\right]_{i, j=1, \ldots, M}$ the so-called mass matrix. Note that this procedure is convenient to easily get a nodal definition of the deformation gradient (but other options are also possible).

\section{Experiments and Results}

\subsection{Data Collection}

Subjects and Image Preparation We selected 4 young adult patients. MRI angiography of the heart were acquired with a $1.5 \mathrm{~T}$ MR scanner (Signa HDx, GE Medical Systems). Images were acquired in the short-axis view covering entirely both ventricles and the pulmonary artery (isotropic in-plane resolution: $0.78 \times 0.78 \mathrm{~mm}^{2}$ to $1.7 \times 1.7 \mathrm{~mm}^{2}$; slice thickness: $2 \mathrm{~mm}$; spacing between slices $1 \mathrm{~mm})$.

Surface Meshes Preparation We segmented the pulmonary artery from MR angiography through image thresholding and manual corrections. The artery was segmented from the pulmonary valve annulus, which connects the artery to the right ventricle, to about $1-2 \mathrm{~cm}$ after the pulmonary branches, which go towards the lungs. From the resulting binary mask, meshes were extracted using marching cubes algorithm. The meshes were then pre-processed for CFD simulations with 3 -MATIC ${ }^{5}$ to impose more easily usable boundary conditions. Inlet and outlets were cut by a plane and extended by approximately $1 \mathrm{~cm}$ (see fig. 2).
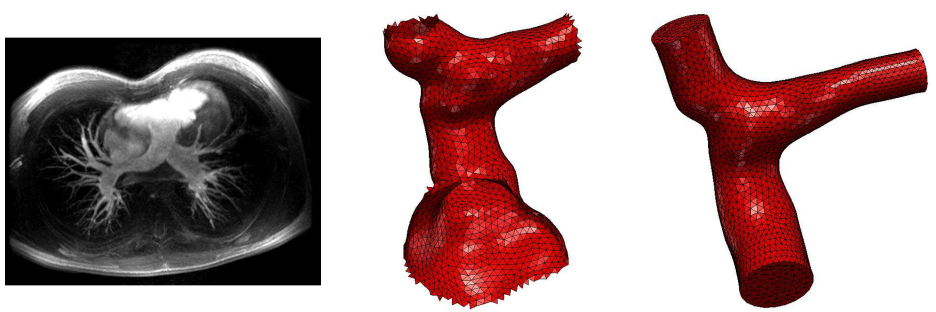

Fig. 2. Left: The raw image before segmentation. Centre: The pre-processed segmented mesh. Right: The same mesh after processing.

\subsection{Statistical Shape Model of the Pulmonary Arteries}

To estimate the template $\widehat{\mathcal{T}}$, two parameters must be set (Sec. 2.1): $\lambda_{V}$, which defines the "stiffness" of the non-linear deformations (larger $\lambda_{V}$ values give more

\footnotetext{
${ }^{5}$ www.materialise.com
} 
global transformations, i.e. rigid body); and $\lambda_{W}$, which characterises the resolution of the currents representation (for lower $\lambda_{W}$ values more subtle shape features can be analysed). As we were mainly interested in the regional ToF alterations (dilation, valve enlargement, regional bulging), these parameters were set to $\lambda_{W}=30 \mathrm{~mm}, \lambda_{V}=5 \mathrm{~mm}$ for the template.

Four iterations of the alternate minimisation for the shape template were needed to reach convergence. The resulting template $\widehat{\mathcal{T}}$ was well centred (mean over standard deviation of the deformations was 0.42575 , see fig. 3). Creation of the template took under 16 hours using parallel implementation of the algorithm on Xeon 2.66Ghz cores. In this way, patient-to-template registration is implemented simultaneously, which means that the template creation is minimally dependent of the number of patients used to create it. The initialized template creation took under 15 minutes, each parallel implementation of the templateto-patient registration took under 15 minutes, and each template update took between $4-6$ hours.

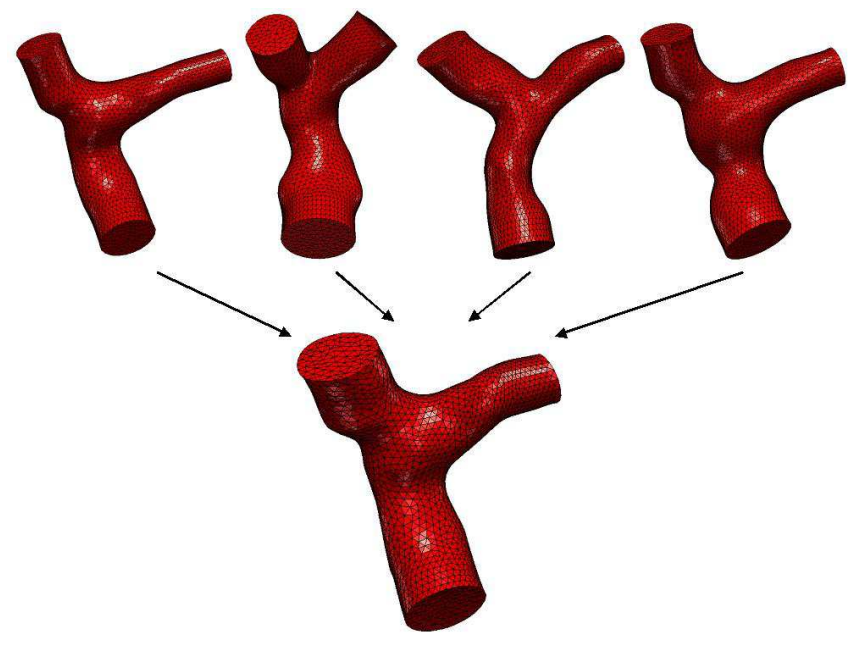

Fig. 3. The four segmented (processed) patient meshes (top row) used to create the template (bottom row).

\subsection{Validation of Atlas-Based Flow Simulations on the Pulmonary Artery}

To verify that the template is unbiased, we created a template with the four patient meshes from this study plus an extra mesh not used in this study, and compared that with template created with four patients. The meshes are shown in fig. 4. As we can see, the templates are very close. The main goal of POD 

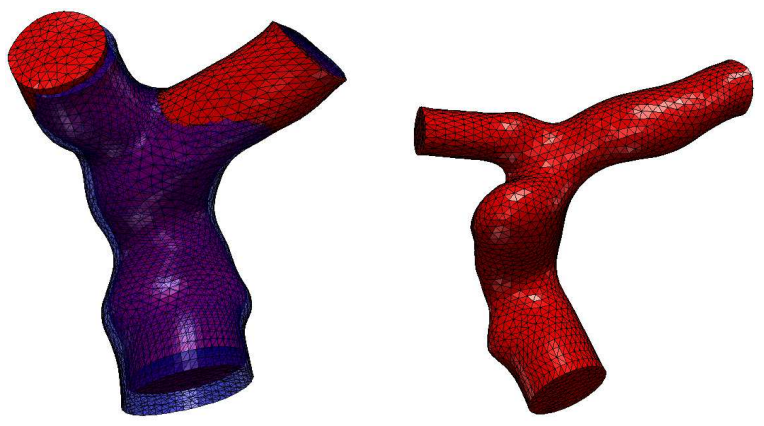

Fig. 4. Left: the template created using five patients (blue) compared to the template created with four patients (red) Right: the extra patient mesh included in the template creation with five patients.

is that, once having reduced the number of degrees of freedom of the discrete problem, one can look for a reduced numerical solution belonging to the subspace spanned by the POD basis. Hence, POD basis on each individual geometry could be extremely useful to perform fast fluid simulations, without the need of solving a full problem on each new patient geometry. However, it is not assured that the basis computed from the template geometry can represent well the fluid solution on a particular shape. Actually, it is even likely that the transported basis is in general unable to represent correctly the solution in the new geometry. Nevertheless, in the case of Tetralogy of Fallot, we may hope that the variability is sufficiently moderate so that the proposed approach is reasonably accurate.

To assess this conjecture, we performed direct numerical simulations of the flow on the template (fig. 5) and on the four particular geometries, with the same boundary conditions. These numerical solutions $\left(\mathbf{u}_{h}, p_{h}\right)$ are then $L^{2}$-projected onto the spaces

$$
U=\operatorname{span}\left\{\phi_{i}^{\mathbf{u}}\right\}_{i=1}^{N_{\mathrm{m}}}, \quad P=\operatorname{span}\left\{\phi_{i}^{p}\right\}_{i=1}^{N_{\mathrm{m}}}
$$

spanned by the transported POD basis (given by (3) and (2)). For comparison purposes, the following quantities were computed:

- instantaneous $L^{2}$-norm difference in velocity and pressure :

$$
\frac{\left\|\mathbf{u}_{h}-\Pi_{U}\left(\mathbf{u}_{h}\right)\right\|_{L^{2}(\Omega)}}{\max _{t}\left\|\mathbf{u}_{h}\right\|_{L^{2}(\Omega)}}, \frac{\left\|p_{h}-\Pi_{P}\left(p_{h}\right)\right\|_{L^{2}(\Omega)}}{\max _{t}\left\|p_{h}\right\|_{L^{2}(\Omega)}} ;
$$

- instantaneous difference in mean inlet and outlet pressures:

$$
\max _{i=1,2}\left\{\frac{\left|p_{h}\right|_{\text {inlet }}-\left.p_{h}\right|_{\text {outlet }_{i}}-\left(\left.\Pi_{P}\left(p_{h}\right)\right|_{\text {inlet }}-\left.\Pi_{P}\left(p_{h}\right)\right|_{\text {outlet }_{i}}\right) \mid}{\left|p_{h}\right|_{\text {inlet }}-\left.p_{h}\right|_{\text {outlet }_{i}} \mid}\right\},
$$

where $\Pi_{U}\left(\right.$ resp. $\left.\Pi_{P}\right)$ stands for the $L^{2}$-projection onto $U$ (resp. $P$ ), given by

$$
\int_{\Omega}\left(\Pi_{U}\left(\mathbf{u}_{h}\right)\right)(\mathbf{x}) \cdot \boldsymbol{\phi}_{i}^{\mathbf{u}}(\mathbf{x}) \mathrm{d} \mathbf{x}=\int_{\Omega} \mathbf{u}_{h}(\mathbf{x}) \cdot \boldsymbol{\phi}_{i}^{\mathbf{u}}(\mathbf{x}) \mathrm{d} \mathbf{x} \quad i=1, \ldots, N_{\mathrm{m}}
$$



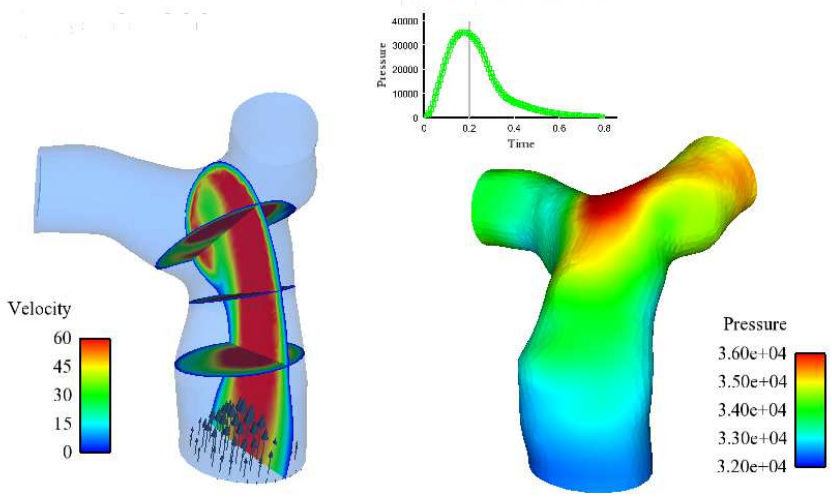

Fig. 5. Flow simulation on the template. Left: velocity (absolute value) on different cutting planes. Right: pressure on the external wall. The curve on top shows the outlet pressure.

and similarly for the pressure.

The length of time to compute the flow simulations were between $15-20$ mins for a blood cycle. The obtained error curves for the four patients are shown in fig. 6. In three of the four cases, we obtained encouraging results. Taking into account a relatively small number of POD basis functions, relative errors for the fluid solution were below $30 \%$. Here, we chose 20 modes for a time dependent solution represented by 100 snapshots, based on the fact that taking more modes did not significantly decrease the error. One shall remark that the worst behaving case was also the one with largest deformation. Similarly, comparing pressure difference we obtained relatively small errors (from $5 \%$ to $20 \%$, for the most deformed geometry).
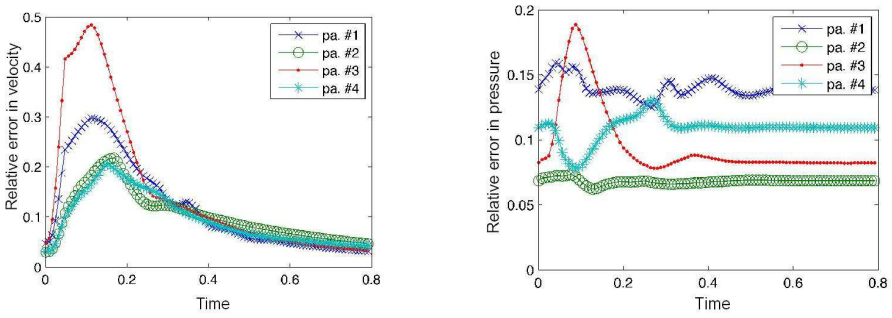

Fig. 6. Error in velocity (norm $L^{2}$ ) and pressure (difference between inlet and outlet) between the full fluid solution on the patient meshes $(1,2,3,4)$ and their projections on the deformed POD basis. 


\section{Discussion and Future Work}

The idea proposed in this paper consists of using an atlas of geometries to build reduced order models based on POD. In this preliminary study, we have only tested the approximation properties of the transported POD bases. Owing to the well-known sensitivity of the flow to the geometry, it was not expected to get a high accuracy for details of the flow. Nevertheless, in the case of Tetralogy of Fallot, we have illustrated that it may be possible to get a reasonable accuracy for some quantities of interest like the pressure drop between inlet and outlet. For the template creation, four iterations were required to satisfy the conditions for convergence, therefore the total time to create the template was lengthy. However, visual inspection of the corresponding meshes showed very small changes along these iterations. In future work it may be interesting to investigate further the best convergence conditions for the template update to optimize the template creation time while preserving the necessary features of the shape for flow simulation. The quality of the results could also be improved by computing the template POD basis for more snapshots, coming from different template geometries and different physiological situations. The transported bases will then be used to actually discretize the model and run reduced order simulations, with patient specific boundary conditions.

\section{References}

1. Durrleman, S., Pennec, X., Trouvé, A., Ayache, N.: A forward model to build unbiased atlases from curves and surfaces. In Pennec, X., Joshi, S., eds.: Proc. of the International Workshop on the Mathematical Foundations of Computational Anatomy (MFCA-2008). (2008)

2. Durrleman, S., Pennec, X., Trouv, A., Ayache, N.: Statistical Models of Sets of Curves and Surfaces based on Currents. Medical Image Analysis (2009) 13(5) (2009) 793-808

3. Vaillant, M., Glaunes, J.: Surface matching via currents. In: Proc. IPMI 2005, Springer (2005) 381

4. Vignon-Clementel, I., Figueroa, C., Jansen, K., Taylor, C.: Outflow boundary conditions for 3D simulations of non-periodic blood flow and pressure fields in deformable arteries. Computer Methods in Biomechanics and Biomedical Engineering (2010) 111(3) (2010) 502-513

5. Wu, C., Liang, Y., Lin, W., Lee, H., Lim, S.: A note on equivalence of proper orthogonal decomposition methods. J. Sound Vibration 265(5) (2003) 1103-1110

6. Bergmann, M., Bruneau, C., Iollo, A.: Enablers for robust POD models. Journal of Computational Physics 228(2) (2009) 516-538

7. Rathinam, M., Petzold, L.: A new look at proper orthogonal decomposition. SIAM Journal on Numerical Analysis 41(5) (2004) 1893-1925

8. Løvgren, A., Maday, Y., Rønquist, E.: The reduced basis element method for fluid flows. In: Analysis and simulation of fluid dynamics. Adv. Math. Fluid Mech. Birkhäuser (2007) 129-154

9. Ciarlet, P.: Mathematical elasticity. Vol. I. Volume 20 of Studies in Mathematics and its Applications. North-Holland Publishing Co. (1988) Three-dimensional elasticity. 